\title{
Stima della portata della fistola artero-venosa mediante termodiluizione nei pazienti in emodialisi cronica: esperienza monocentrica
}

\author{
Enrico Varricchio ${ }^{1}$, Alessandro Puntoni ${ }^{1}$, Domenico Giannese ${ }^{2}$, Claudia Mannucci ${ }^{1}$, Piera Serio ${ }^{1}$, Raffaele Caprioli ${ }^{2}$, \\ Alberto Lippi ${ }^{2}$, Maria Francesca Egidi' ${ }^{2}$, Adamasco Cupisti ${ }^{1}$ \\ ${ }^{1}$ Dipartimento di Medicina Clinica e Sperimentale, Università di Pisa, Pisa - Italia \\ ${ }^{2}$ U.O. Nefrologia Trapianti e Dialisi, AOUP, Pisa - Italia
}

\begin{abstract}
Flow estimate of arteriovenous fistula by thermodilution in patients on chronic hemodialysis: monocentric experience

Purpose: Vascular access surveillance in hemodialysis is today an important challenge for nephrologist. Low blood flow is a risk factor for development of thrombosis of native fistula or graft.

The aim of the study is to evaluate the correspondence between flow measurements by thermodilution and Color Doppler, and to identify flow rate values using the Blood Temperature Monitor (BTM) method in the case of stenosis.

Methods: We evaluated 29 patients on chronic hemodialysis. The evaluations of blood flow with BTM were performed during the first hour of the hemodialysis session. All patients underwent a Color Doppler of vascular access within 10 days from the BTM measurements.

Results: The mean vascular access flow calculated with BTM resulted $1142 \pm 700 \mathrm{~mL} / \mathrm{min}$ and there was a correlation with Color Doppler data $1199 \pm 644 \mathrm{~mL} / \mathrm{min}(p=0,0001 \mathrm{r}=0,829)$. The flow of patients with hemodynamically significant stenosis was $332 \pm 92 \mathrm{~mL} / \mathrm{min}$ with a minimum value of $270 \mathrm{~mL} / \mathrm{min}$ and a maximum value of $440 \mathrm{~mL} / \mathrm{min}$. Conclusions: The correspondence between thermodilution and Color Doppler supports the implementation of intradialitic evaluation of the vascular access blood flow with the BTM method. Thermodilution allows to identify earlier patients with high risk of vascular access failure and the need for further diagnostic and therapeutic investigations.
\end{abstract}

Keywords: Arterovenous fistula, Blood Temperature Monitor, Hemodialysis, Stenosis, Thermodilution

\section{Introduzione}

La sorveglianza dell'accesso vascolare è ad oggi un'importante sfida per il nefrologo in emodialisi. II malfunzionamento dell'accesso vascolare riduce l'efficienza dell'emodialisi e la bassa portata è un fattore di rischio per lo sviluppo di trombosi e la definitiva perdita dell'accesso vascolare $(1,2)$. La salvaguardia di una fistola artero-venosa (FAV) ben funzionante

Received: January 08, 2020

Accepted: January 20, 2020

Published online: March 09, 2020

Indirizzo per la corrispondenza:

Enrico Varricchio

Dipartimento di Medicina Clinica e Sperimentale

Università di Pisa

Via Roma 67

56126 Pisa- Italia

enricobn@alice.it mediante il costante monitoraggio del flusso è una premessa essenziale per raggiungere l'adeguatezza dialitica e il mantenimento della FAV.

Le Linee Guida 2006 della National Kidney Foundation Kidney Disease Outcomes Quality Initiative (NKF KDOQI) indicano che una sorveglianza della fistola, sia essa nativa o protesica, può permettere in caso di stenosi un tempestivo intervento correttivo e migliorare così la pervietà del vaso $\mathrm{e}$ diminuire l'incidenza dell'ostruzione. Le stenosi emodinamicamente significative infatti riducono il flusso, aumentano le turbolenze nel lume e spingono le piastrine contro la parete del vaso, favorendone l'attivazione (3).

Oltre alle tecniche dirette come l'Eco-Color-Doppler (ECD) ci sono anche tecniche indirette di misurazione del flusso della FAV che possono essere eseguite direttamente al letto del paziente durante il trattamento emodialitico.

Le tecniche impiegate sono la termodiluizione, la diluizione a ultrasuoni, la dialysance ionica e l'otticodiluizione. Tutte queste si basano sulla formula introdotta da Krivitski, che prevede la stima del flusso dell'accesso vascolare 
invertendo le linee ematiche arteriosa e venosa durante il trattamento (4). A oggi, il metodo di riferimento per la misurazione del flusso durante l'emodialisi è rappresentato dalla diluizione a ultrasuoni. Comunque i dati di Lopot dimostrano che il ricircolo ottenuto con termodiluizione è un metodo alternativo ed efficace nella valutazione del flusso e l'affidabilità del test può essere aumentata raddoppiando il numero di misurazioni (5).

La misurazione con termodiluizione mediante il sistema "Blood Temperature Monitor" (BTM) si ottiene riducendo la temperatura del dialisato di circa $2,5-3^{\circ}$ per circa 5 minuti, per cui si riduce anche la temperatura del sangue che ritorna nel versante venoso; la quota di sangue a minore temperatura viene poi rilevata da un sensore termico per ottenere la stima del ricircolo della FAV. II ricircolo ottenuto con BTM include anche la quota del ricircolo cardiopolmonare, che può essere, però, annullato se vengono presi in considerazione sia i valori di ricircolo ottenuti con linee in posizione normale sia quelli ottenuti con linee in posizione invertita $(2,6)$.

Le Linee Guida KDOQI per gli accessi vascolari raccomandano una sorveglianza almeno mensile con le attuali tecniche di misurazione del flusso e di valutare un'ulteriore indagine con fistolografia se la portata scende sotto i $600 \mathrm{~mL} / \mathrm{min}$ nei graft e sotto i $500 \mathrm{~mL} / \mathrm{min}$ nelle FAV native o se la portata ha un decremento maggiore del $25 \%$ in quattro mesi, ponendo l'accento sull'importanza del monitoraggio e dell'andamento del flusso nel tempo.

Le tecniche di misurazione del flusso sia dirette che indirette, non sono mutuamente esclusive (3). L'ECD offre numerosi vantaggi perché è in grado di eseguire anche una valutazione anatomica dei vasi e consente di vedere anche le stenosi iniziali che difficilmente causano riduzione di flusso e di individuare la sede di eventuali stenosi. L'ECD può anche chiarire la causa della stenosi (iperplasia intimale, trombi parietali, cicatrici in sede di venipuntura, ecc.) $(7,8)$. Inoltre l'ECD rispetto all'angiografia ha minori costi e minore invasività e non utilizza il mezzo di contrasto iodato.

L'obiettivo di questo studio è quello di stimare la corrispondenza tra la misurazione del flusso con BTM e con ECD e di individuare valori di portata calcolata con la metodica BTM (QaBTM) corrispondenti a una stenosi emodinamicamente significativa.

\section{Metodi}

Lo studio è monocentrico e osservazionale e include pazienti con età $>18$ anni, in emodialisi cronica e portatori di FAV nativa o protesica, clinicamente stabili da almeno 3 mesi.

Sono stati esclusi dallo studio i pazienti in emodialisi portatori di catetere venoso centrale o in dialisi eseguita con monoago e i pazienti con infezioni dell'accesso vascolare in atto o che non hanno fornito il consenso alle procedure previste dallo studio.

Della popolazione di 111 pazienti in emodialisi, sono stati selezionati 29 pazienti (10F e $19 \mathrm{M})$ ), età $62 \pm 13$ anni, età dialitica $41 \pm 36$ mesi, 6 portatori di FAV protesica e 23 portatori di FAV nativa. Per ogni paziente sono stati riportati peso di inizio e di fine dialisi e i parametri ematochimici, emoglobina, albuminemia a inizio dialisi e Kt/V. Inoltre, sono stati valutati i seguenti parametri intradialitici: distanza in centimetri tra i due punti di incannulazione della fistola, pressione arteriosa e pressione venosa del circuito, flusso sangue $(\mathrm{Qb})$ e ricircolo eseguito con "Blood Temperature Monitor" (RBTM) su dispositivi Fresenius 5008 con linea arteriosa e venosa in posizione normale e con linea arteriosa e venosa in posizione invertita. II Kt/V è stato calcolato mediante Online Clearance Monitoring (OCM) presente sul dispositivo Fresenius 5008 (Tab. I).

Tabella I - Parametri ematochimici e dialitici dei pazienti valutati nello studio

\begin{tabular}{lcc}
\hline & Media \pm DS & Mediana (IQR) \\
\hline Emoglobina (g/dL) & $10,3 \pm 1,2$ & $10,3(1,5)$ \\
Albuminemia (g/dL) & $3,9 \pm 0,2$ & $4(0,4)$ \\
Ricircolo linee posizione normale (\%) & $11 \pm 4(6)$ \\
Ricircolo linee posizione invertita (\%) & $32 \pm 11$ & $30(14)$ \\
Flusso sangue prescritto (Qb) mL/min & $285 \pm 36$ & $300(0)$ \\
Ricircolo cardiopolmonare (\%) & $30 \pm 14$ & $27(24)$ \\
Pressione arteriosa linee posizione normale $(\mathrm{mmHg})$ & $-124 \pm 35$ & $-132(44)$ \\
Pressione venosa linee posizione normale $(\mathrm{mmHg})$ & $127 \pm 24$ & $125(20)$ \\
Pressione arteriosa linee posizione invertita $(\mathrm{mmHg})$ & $-146 \pm 33$ & $-157(31)$ \\
Pressione venosa linee posizione invertita $(\mathrm{mmHg})$ & $136 \pm 25$ & $135(15)$ \\
Kt/V & $1,21 \pm 0,19$ & $1,26(0,3)$ \\
Incremento ponderale interdialitico $(\mathrm{kg})$ & $2,6 \pm 1$ & $1,8(1,5)$ \\
Distanza aghi (cm) & $6,3 \pm 2,8$ & $5(2,75)$ \\
\hline
\end{tabular}


Il calcolo della portata dell'accesso vascolare e del ricircolo cardiopolmonare misurati tramite la percentuale del ricircolo ottenuto con il BTM sono stati eseguiti con le seguenti formule (6):

$$
\begin{aligned}
& \text { QabTM }=\frac{1-\text { RBTMx }}{R B T M x(1-\text { CPRbtm })} \times Q b x \\
& \text { CPRbtm }=\frac{\text { RBTMn }(1-\text { RBTMx })}{R B T M x(1-\text { RBTMn })} \times \frac{Q b x}{Q b n}
\end{aligned}
$$

Dove il QaBTM è la portata dell'accesso vascolare calcolata con BTM, il CPRbtm è il ricircolo cardiopolmonare calcolato con BTM, il RBTMn è il ricircolo calcolato con linee in posizione normale, il RBTMx è il ricircolo calcolato con linee in posizione invertita, il Qbn è il flusso sangue con linee in posizione normale e il Qbx è il flusso sangue con linee in posizione invertita. I parametri necessari per il calcolo della portata dell'accesso vascolare (QaBTM) sono stati rilevati durante la prima ora della seduta emodialitica. Il calcolo è stato eseguito tramite il BTM, considerando il ricircolo e il Qb su linee arteriosa e venosa in posizione sia normale che invertita. La valutazione è stata ripetuta due volte e il dato finale è stato calcolato con la media dei due risultati ottenuti tramite la formula descritta precedentemente.

Tutti i pazienti sono stati poi inviati a eseguire un ECD dell'accesso vascolare in un giorno interdialitico entro massimo 10 giorni dalle misurazioni eseguite in dialisi. La portata della FAV all'ECD è stata misurata a livello dell'arteria brachiale e tutte le misurazioni sono state eseguite in cieco da due radiologi esperti. Sono state valutate le relazioni tra la misurazione del flusso dell'accesso vascolare sia con BTM che con ECD e l'entità del ricircolo, l'efficacia dialitica (Kt/V) e i valori pressori del circuito.

Dei 29 pazienti selezionati per lo studio sono stati analizzati i dati di 26, poiché in 3 pazienti il calcolo del QaBTM non era affidabile: in particolare, sono stati esclusi dallo studio i pazienti con abnormi valori di portata stimata superiori a $5.000 \mathrm{~mL} / \mathrm{min}$.

Dei 26 pazienti esaminati, in 13 i dati documentavano una stenosi all'ECD e del gruppo dei pazienti con stenosi 4 avevano una stenosi emodinamicamente significativa.

La diagnosi di stenosi emodinamicamente significativa all'ECD è stata formulata in base alla presenza dei due criteri principali e di almeno un criterio addizionale.

\section{Criteri principali:}

- $\quad$ Riduzione del lume vascolare $>50 \%$

- PSV ratio: rapporto tra il PSV nel tratto stenotico e il PSV nel tratto pre-stenotico. Si considera diagnostico un valore $>2$.

Criteri addizionali:

- Calcolo della portata a livello dell'arteria omerale. Si considera diagnostico un Qa $<500 \mathrm{~mL} / \mathrm{min}$ su FAV native o $<600 \mathrm{~mL} / \mathrm{min}$ su FAV protesiche

- $\quad$ Diametro residuo del vaso $<2 \mathrm{~mm}$ (7).
Sulla base dei referti dell'ECD i pazienti sono stati divisi in tre gruppi in base alla segnalazione e alla significatività delle stenosi documentate con la metodica ECD.

I tre gruppi erano caratterizzati da: assenza di stenosi (AS), stenosi non emodinamicamente significativa (NES) e stenosi emodinamicamente significativa (ES).

Tra questi sono state ricercate eventuali differenze in termini di ricircolo, QaBTM, Qb, Kt/V e portata calcolata sull'arteria brachiale con l'ECD.

I risultati sono riportati come media \pm deviazione standard. La correlazione lineare di Pearson è stata usata per analizzare la correlazione tra i valori della portata della FAV ottenuti con il BTM e quelli ottenuti con ECD. Per il confronto tra i pazienti divisi secondo presenza e significatività della stenosi sono stati impiegati i seguenti test statistici: ANOVA one way e t-test per le variabili continue. È stato considerato statisticamente significativo il valore di $p$ inferiore a 0,05.

\section{Risultati}

La portata dell'accesso vascolare calcolata con BTM è stata di $1.142 \pm 700 \mathrm{~mL} / \mathrm{min}$, simile alla portata dell'accesso vascolare calcolata con la metodica ECD $1.199 \pm 644 \mathrm{~mL} / \mathrm{min}$.

Esiste una buona corrispondenza tra le due metodiche, come mostrato nella Figura 1.

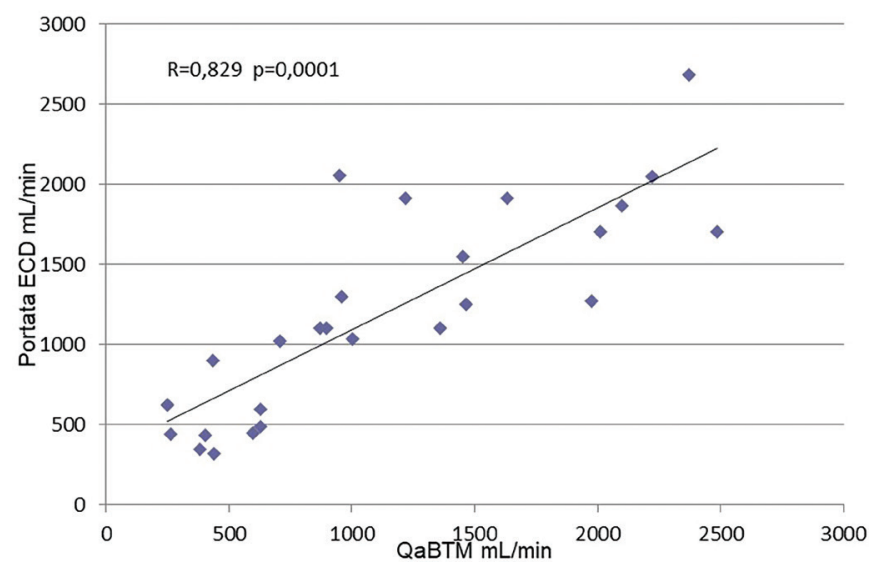

Fig. 1 - Correlazione lineare tra la portata calcolata con BTM (QaBTM) e la portata calcolata con Eco-Color-Doppler (portata ECD).

Nella Tabella II sono riportati i dati relativi ai pazienti dei 3 gruppi: assenza di stenosi (AS), stenosi non emodicamicamente significativa (NES) e stenosi emodinamicamente significativa (ES). Esiste una significatività statistica tra i gruppi per il flusso calcolato con ECD, per il QaBTM, per il ricircolo a linee invertite, per il $\mathrm{Qb}$ e per il Kt/V (Fig. 2).

In particolare, i soggetti con stenosi emodinamicamente significativa avevano valori di portata all'ECD e di QaBTM inferiori sia rispetto ai soggetti con stenosi non emodinamicamente significativa sia rispetto a quelli senza stenosi. II ricircolo a linee invertite dei soggetti con stenosi emodinamicamente significativa era superiore sia rispetto ai soggetti con stenosi non emodinamicamente significativa sia rispetto a quelli senza 
TABELLA II - Parametri valutati nei pazienti senza stenosi, con stenosi non emodinamicamente significativa e con stenosi emodinamicamente significativa della fistola artero-venosa o graft all'Eco-Color-Doppler

\begin{tabular}{|c|c|c|c|c|}
\hline Stenosi & Assente & $\begin{array}{c}\text { Emodinamicamente } \\
\text { non significativa }\end{array}$ & $\begin{array}{l}\text { Emodinamicamente } \\
\text { significativa }\end{array}$ & $\mathbf{p}$ \\
\hline Flusso Eco-Color-Doppler (mL/min) & $1449 \pm 600$ & $1179 \pm 594$ & $432 \pm 135 b^{*}$ & 0,015 \\
\hline Ricircolo linee normali (\%) & $13 \pm 4$ & $9 \pm 3$ & $10 \pm 5$ & 0,09 \\
\hline Ricircolo linee invertite (\%) & $30 \pm 10$ & $28 \pm 8$ & $46 \pm 7 b^{* *}$ & 0,0061 \\
\hline Pressione arteriosa linee normali ( $\mathrm{mmHg}$ ) & $125 \pm 28$ & $129 \pm 40$ & $110 \pm 37$ & 0,65 \\
\hline Pressione venosa linee normali (mmHg) & $126 \pm 19$ & $129 \pm 22$ & $122 \pm 43$ & 0,89 \\
\hline Pressione arteriosa linee invertite $(\mathrm{mmHg})$ & $160 \pm 18$ & $162 \pm 20$ & $117 \pm 40$ & 0,05 \\
\hline
\end{tabular}

$a=p<0,05, b=p<0,01$ vs AS, ${ }^{*}=p<0,05,{ }^{* *}=p<0,01$ vs NES

\section{QaBTM}

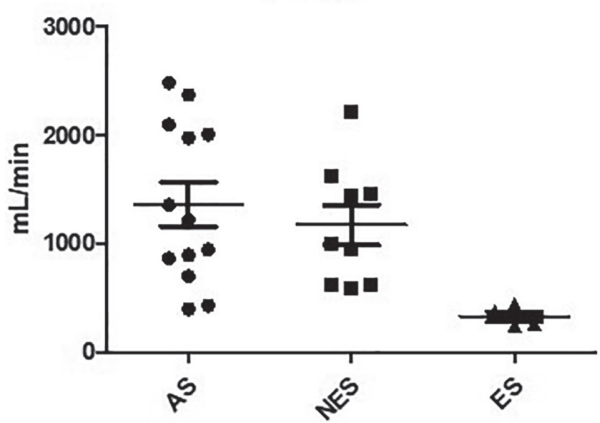

Ricircolo linee invertite

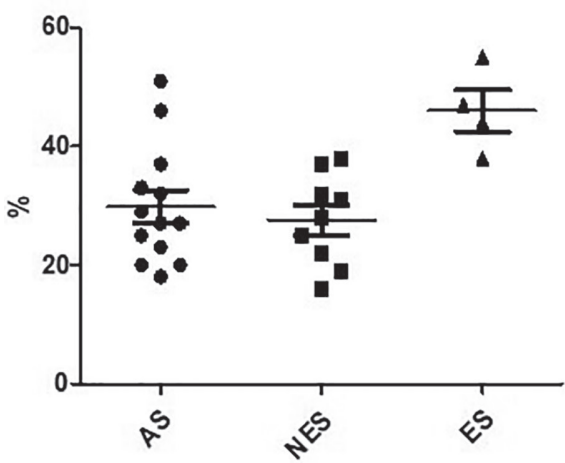

Portata ECD

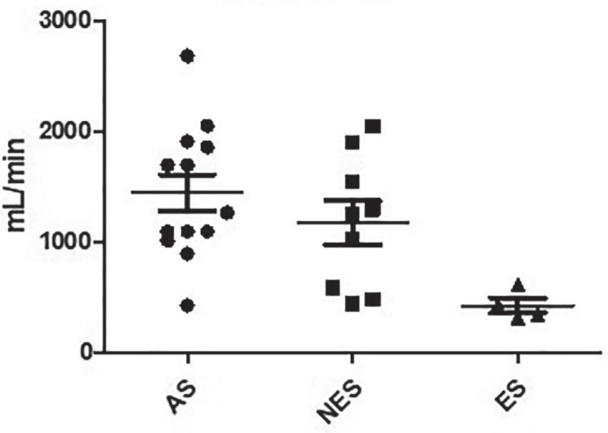

$\mathrm{Kt} / \mathrm{V}$

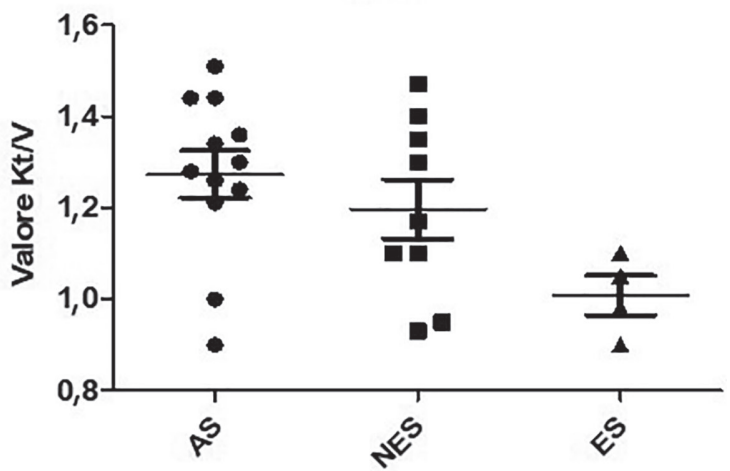

Fig. 2 - Portata calcolata con BTM (QaBTM), portata calcolata all'Eco-Color-Doppler (portata ECD), ricircolo calcolato a linee invertite e Kt/V nei pazienti divisi in base all'assenza di stenosi (AS), alla presenza di stenosi non emodinamicamente significativa (NES) e stenosi emodinamicamente significativa (ES) 
stenosi. Inoltre i soggetti con stenosi emodinamicamente significativa avevano un Kt/V inferiore ai soggetti senza stenosi.

Abbiamo poi confrontato il gruppo AS con il gruppo NES.

Nei 13 pazienti senza stenosi all'ECD la portata calcolata con BTM era di $1368 \pm 735 \mathrm{~mL} / \mathrm{min}$ e con ECD di $1449 \pm 600$ $\mathrm{mL} / \mathrm{min}$. Nei 9 pazienti con stenosi non emodinamicamente significativa la portata calcolata con BTM era di $1175 \pm 555$ $\mathrm{mL} / \mathrm{min}$ e quella calcolata con ECD di $1179 \pm 594 \mathrm{~mL} / \mathrm{min}$.

Non sono state rilevate differenze statisticamente significative in termini di portata tra i due gruppi per nessuna delle due metodiche prese in considerazione.

Inoltre dai nostri dati non emerge nessuna differenza statisticamente significativa neanche per gli altri parametri dialitici, come il Kt/V, il ricircolo a linee invertite e il Qb.

\section{Discussione}

I risultati di questo studio confermano che i valori di flusso della FAV calcolati mediante BTM sono sovrapponibili a quelli calcolati con ECD.

Il coefficiente di correlazione tra le due metodiche emerso dai nostri dati ( $R=0,829 ; p=0,0001)$ è in linea con quello di altri studi presenti in letteratura, in particolare quello del gruppo francese di Mathieu Sacquépée $\left(R^{2}=0,86\right)(9)$ e quello di Marques $(R=0,851)(8)$.

II BTM offre informazioni preziose al nefrologo non solo perché consente di calcolare il flusso dell'accesso vascolare, ma anche perché il calcolo del ricircolo può dare informazioni importanti sulla pervietà della fistola. Tuttavia, il ricircolo calcolato a linee in posizione normale non sempre risulta alterato in presenza di stenosi. Infatti, dai nostri dati si evince che solo il ricircolo misurato a linee invertite è aumentato nei pazienti con stenosi emodinamicamente significativa con un valore medio del $46 \%$.

Una spiegazione di questo fenomeno è stata fornita da Schneditz, secondo cui, negli accessi con stenosi tra il sito di puntura arterioso e venoso, il flusso bypassa la stenosi attraverso la circolazione extracorporea. Quindi, in tal caso, non è possibile rilevare un aumento del ricircolo, se misurato a linee in posizione normale. Invece con linee in posizione invertita, il flusso sanguigno forzato attraverso la stenosi si adegua alle resistenze offerte dal vaso, per cui il ricircolo avrà un valore più alto (6). In ogni caso, misurazioni seriate nel tempo permettono anche di monitorare l'andamento del Qa rispetto a una singola misurazione.

Le Linee Guida KDOQI sugli accessi vascolari del 2006 suggerivano ulteriori indagini per rilevare la presenza di stenosi quando si riscontra un decremento del Qa maggiore del $25 \%$ rispetto a un valore basale di $1.000 \mathrm{~mL} / \mathrm{min}$.

Attualmente c'è molto dibattito in letteratura sulla determinazione di un valore soglia che possa essere predittivo di stenosi emodinamicamente significative.

Nel nostro studio, il QaBTM dei pazienti con ES è stato di $332 \pm 92 \mathrm{~mL} / \mathrm{min}$, con un valore minimo di $270 \mathrm{~mL} / \mathrm{min}$ e un valore massimo di $440 \mathrm{~mL} / \mathrm{min}$ e tutti avevano delle stenosi $>60 \%$ all'ECD. Un recente studio del 2018 identificava un Qa $<583 \mathrm{~mL} / \mathrm{min}$ nelle FAV distali come valore soglia ottimale nel predire stenosi (10) e Tessitore et al identificavano un Qa $<750 \mathrm{~mL} / \mathrm{min}$ calcolato con diluizione a ultrasuoni (11). In una review della letteratura del 2014, Tessitore (12) mostrava che, nelle fistole, un Qa $<900 \mathrm{~mL} / \mathrm{min}$ aveva una sensibilità dell' $88 \%$ e un valore predittivo positivo del $66 \%$ nel rilevare stenosi. Se la soglia di Qa invece era $<500 \mathrm{~mL} / \mathrm{min}$ la sensibilità era del 59\% e il valore predittivo positivo dell' $83 \%$.

In 9 pazienti l'ECD segnalava una stenosi non emodinamicamente significativa, in quanto non erano soddisfatti tutti i criteri descritti prima. In questi pazienti erano riportate stenosi del 50\% circa e, nonostante sia il QaBTM che la portata rilevata all'ECD fossero lievemente più bassi rispetto a quelli dei pazienti che non avevano stenosi, non sono state rilevate differenze tra questi due gruppi.

La termodiluizione può essere impiegata con successo per il monitoraggio della FAV in dialisi. II BTM, rispetto al Doppler, non ha costi aggiuntivi, non è operatore-dipendente, si può eseguire durante il trattamento emodialitico senza prolungare il tempo di transito del paziente in ospedale e non richiede attrezzature specifiche che non siano già integrate in alcuni monitor di dialisi. Va inoltre segnalato che la procedura di calcolo del ricircolo a linee invertite è oggi facilitata dall'uso del Twister, un device che consente di invertire le linee del paziente senza disconnetterle manualmente (10).

In ogni caso, il BTM non è sostituibile all'ECD nel rilevamento delle iniziali stenosi che non danno ancora alterazioni significative di flusso e nella definizione della sede e dell'entità del problema. L'ECD infatti caratterizza la stenosi e il suo impatto sul flusso e le ultime Linee Guida spagnole del 2017 raccomandano l'utilizzo dell'ECD come primo esame di imaging, anche senza conferma fistolografica, per porre indicazione di angioplastica (PTA) elettiva, se è presente una stenosi emodinamicamente significativa. D'altro canto, l'ECD è operatore-dipendente e richiede personale addestrato che non sempre è reperibile nei centri dialisi. Invece la fistolografia, nonostante rappresenti il gold standard nella diagnosi di stenosi, è raccomandata nel caso in cui l'indagine ecografica non sia diagnostica e se persiste il sospetto di stenosi emodinamicamente significativa (13). II principale limite dello studio è rappresentato dal piccolo numero dei pazienti provenienti da un unico centro. Inoltre, anche se l'ECD è stato eseguito a distanza di pochi giorni dalla misurazione del flusso in dialisi, non possiamo eliminare completamente le differenze dovute al diverso stato di idratazione.

Nel complesso, il nostro studio conferma l'utilità del QaBTM come primo approccio diagnostico che però non è mirato a sostituire, ma, piuttosto, ad integrare le tecniche dirette di misurazione del flusso. Questo consente di definire la tempistica della sorveglianza dell'accesso vascolare e favorisce una migliore selezione dei pazienti che necessitano di ulteriori indagini.

\section{Disclosures}

Financial support: The authors declare no financial support either from public, private or charity organisations.

Conflict of interest: The authors declare no conflict of interest.

\section{Bibliografia}

1. Badr B, Bories $\mathrm{P}$, Marais $\mathrm{P}$, et al. Transonic, thermodilution, or ionic dialysance to manage vascular access: Which method is best? Hemodial Int. 2014;18(1):127-35. 
2. Schneditz D, Kaufman AM, Levin N. Surveillance of Access Function by the Blood Temperature Monitor. Semin Dial. 2003;16(6):483-7.

3. Vascular Access Work Group. Clinical practice guidelines for vascular access. Am J Kidney Dis. 2006;48 Suppl 1:S248-73.

4. Krivitski NM. Theory and validation of access flow measurement by dilution technique during hemodialysis. Kidney Int. 1995; 48(1):244-50.

5. Lopot F, Nejedlý B, Sulková S, Bláha J. Comparison of different techniques of hemodialysis vascular access flow evaluation. J Vasc Access. 2004;5(1):25-32.

6. Schneditz D, Wang E, Levin NW. Validation of haemodialysis recirculation and access blood flow measured by thermodilution. Nephrol Dial Transplant. 1999;14(2):376-83.

7. Malik J, Kudlicka J, Novakova L, Adamec J, Malikova H, Kavan J. Surveillance of arteriovenous accesses with the use of duplex Doppler ultrasonography. J Vasc Access. 2014;15 Suppl 7:S28-32.

8. Guedes Marques M, Ibeas J, Botelho C, Maia P, Ponce P. Doppler ultrasound: a powerful tool for vascular access surveillance. Semin Dial. 2015;28(2):206-10.
9. Sacquépée $M$, Tivollier JM, Doussy $Y$, Quirin $N$, Valéry JC, Cantin JF. Comparison of different techniques of hemodialysis vascular access flow evaluation: blood temperature monitoring thermodilution and doppler debimetry. Nephrol Ther. 2012; 8(2):96-100.

10. Choi YJ, Lee YK, Park HC, et al. Prediction of vascular access stenosis: Blood Temperature Monitoring with the Twister versus static intra-access pressure ratio. PLos One. 2018;13(10): e0204630.

11. Tessitore N, Bedogna V, Gammaro L, et al. Diagnostic accuracy of ultrasound dilution access blood flow measurement in detecting stenosis and predicting thrombosis in native forearm arterovenous fistulae for hemodialysis. Am J Kidney Dis. 2003; 42(2):331-41.

12. Tessitore N, Bedogna V, Verlato G, Poli A. Clinical access assessment. J Vasc Access. 2014;15 Suppl 7:S20-7.

13. Ibeas J, Roca-Tey R, Vallespín J, et al. Spanish Clinical Guidelines on Vascular Access for Haemodialysis. Nefrologia. 2017;37 Suppl 1:1-191. 